\title{
Du vinyle à YouTube : les habitudes de consommation et de recherche de musique des jeunes adultes québécois
}

Ariane Legault-Venne

Spécialiste en moyens et techniques d'enseignement

Cégep du Vieux Montréal

alegaultvenne@cvm.qc.ca

Audrey Laplante

Professeure agrégée à l'École de bibliothéconomie et des sciences de l'information Université de Montréal

audrey.laplante@umontreal.ca

Sébastien Leblanc-Proulx

Bibliothécaire

Université du Québec à Chicoutimi

sebastien.leblanc-proulx@uqac.ca

Dominic Forest

Professeur agrégé à l'École de bibliothéconomie et des sciences de l'information Université de Montréal

dominic.forest@umontreal.ca

\section{Résumé}

Il existe peu d'études récentes sur les habitudes de consommation et le comportement dans la recherche d'information musicale à des fins de loisir au Québec et au Canada. Pourtant, les études réalisées sur le sujet dans d'autres pays suggèrent que l'évolution rapide des technologies a entraîné des changements importants dans ces pratiques. II est essentiel d'avoir une bonne connaissance de ces nouvelles pratiques pour concevoir des systèmes de repérage et d'exploration de la musique qui répondent aux besoins réels de leurs utilisateurs. Cet article présente les résultats d'une enquête menée auprès de 278 personnes âgées de 18 à 35 ans habitant au Québec. Les objectifs de l'enquête étaient de mieux connaître les habitudes d'achat et d'écoute de musique des jeunes adultes québécois ainsi que la façon dont ils s'y prennent pour découvrir de nouveaux artistes et de nouvelles musiques. Les résultats révèlent que les services d'écoute de musique en continu et les sites de partage de vidéos étaient utilisés par $78 \%$ des répondants. Malgré la popularité croissante de ces services, les jeunes Québécois n'ont pas totalement délaissé certaines pratiques plus traditionnelles. Ils continuent de valoriser les recommandations musicales de leurs proches, d'écouter de la musique sur CD et d'acheter des fichiers musicaux, même si ces pratiques sont de moins en moins fréquentes. 


\begin{abstract}
Very few recent studies have focused on Quebeckers' and Canadians' consumption and information-seeking behaviour of music for leisure. However, studies conducted in other countries suggest that the rapid evolution of technologies has resulted in important changes in practices. It is essential to gain a better knowledge of these new practices to design music information retrieval and exploration systems that meet the real needs of users. This article presents the results of a survey of 278 younger adults aged 18 to 35 living in Quebec. The objective of the survey was to increase knowledge of the music listening and purchasing behaviour of young music lovers, and to better understand how they discover new artists and new music. Results reveal that music streaming services and video-sharing websites were used by $78 \%$ of respondents. Despite the increasing popularity of these services, young Quebeckers have not entirely abandoned past practices. They still value music recommendations from friends, colleagues or relatives, listen to CDs, and purchase music files, even though these practices are becoming less frequent.
\end{abstract}

\title{
Mots-clés
}

comportement informationnel, musique, études d'utilisateurs, services d'écoute de musique en ligne, recherche d'information musicale

\section{Keywords}

information behaviour, music, user studies, music streaming services, music information retrieval

\section{Introduction}

Le virage à l'ère numérique dans le domaine de la musique a profondément modifié notre rapport à la musique. Autrefois seulement sur support physique, la musique enregistrée peut maintenant être écoutée, partagée, discutée et recommandée en ligne. Les avantages pour les amateurs de musique sont multiples, mais encore faut-il s'approprier les technologies numériques sur lesquelles elles reposent. Toutefois, modifier des habitudes ne se fait pas toujours en un clin d'œil. Quinze ans après l'arrivée de Napster, qu'en est-il de nos pratiques?

Afin de mieux comprendre comment les jeunes adultes découvrent et acquièrent de la musique et pour connaître leurs habitudes d'écoute, nous avons réalisé une enquête auprès de jeunes Québécois âgés de 18 à 35 ans. Plus précisément, nous avons cherché à mieux comprendre la place qu'occupent les nouvelles technologies et les nouveaux services musicaux sur le Web dans leur recherche et leur exploration de musique. 


\section{Études connexes}

Bien que de nombreux efforts soient alloués à développer des systèmes de repérage et de recommandation pour la musique, la façon dont les personnes s'y prennent pour découvrir de la musique à des fins de loisir demeure peu étudiée. En outre, le paysage des systèmes de recherche et d'écoute de musique en ligne change constamment, de sorte que de nouvelles études doivent être réalisées régulièrement.

Quelques études sur le comportement dans la recherche d'information ont néanmoins été réalisées et nous donnent des indications sur la façon dont les gens s'y prennent pour trouver de la musique à des fins de loisir. Des études qualitatives, basées sur des entretiens en profondeur ou sur des observations directes, ont permis de décrire et de comprendre les stratégies qu'emploient les gens pour découvrir de la musique ainsi que leurs motivations (Cunningham, Reeves et Britland, 2003; Laplante, 2008). Quelques études quantitatives ont également été menées pour connaître les besoins et les pratiques de recherche d'information musicale des jeunes adultes (Hu, Lee et Wong, 2014; Lee, Cho et Kim, 2016; Lee et Downie, 2004; Lee et Waterman, 2012; Tepper et Hargittai, 2009).

Ces études ont révélé que découvrir de la musique est souvent une activité sociale. Les gens vont couramment chez le disquaire entre amis ou en couple (Cunningham et al., 2003). Même depuis l'apparition des services de recommandations musicales, les jeunes adultes continuent de découvrir de nouveaux artistes musicaux principalement par l'intermédiaire de leurs amis, parents et collègues (Laplante, 2008; Lee et al., 2016; Lee et Downie, 2004; Tepper et Hargittai, 2009). Les réseaux sociaux sont d'ailleurs devenus des outils privilégiés pour partager des découvertes musicales entre adolescents (Laplante, 2015). Par ailleurs, les jeunes adultes disent faire peu confiance aux professionnels tels que les disquaires ou les critiques musicaux, mais valorisent au contraire le jugement des personnes de leur entourage qui les connaissent bien et peuvent fournir des recommandations personnalisées (Laplante, 2008, 2012; Laplante et Downie, 2006; Lee et al., 2016; Lee et Downie, 2004). Les critiques et évaluations des autres utilisateurs sont aussi consultées par plusieurs, soit par $54,4 \%$ selon l'enquête de Lee (2016). II faut cependant préciser que le fait qu'on se fie peu aux recommandations des services d'écoute de musique en continu ne signifie pas pour autant que ces services ne sont pas utilisés : les études montrent que les jeunes adultes connaissent et utilisent souvent plusieurs sources d'information et services musicaux en ligne, chacun de ces services ou sources étant utilisé dans un objectif précis (p. ex. : écouter de la musique, lire sur la musique ou les artistes) (Hu et al., 2014; Laplante, 2008; Lee et Waterman, 2012).

Que ce soit chez le disquaire ou en ligne, les études qualitatives montrent que les gens utilisent une variété de métadonnées ( $p$. ex. : noms des contributeurs, maison de disque, apparence visuelle de l'album) pour faire des inférences sur l'expérience musicale proposée par un artiste afin de faire un premier tri et d'éviter ainsi d'écouter tout ce qu'ils trouvent. Le genre musical, qui permet de délimiter le secteur à explorer, est le critère le plus souvent utilisé pour faire ce premier tri (Laplante, 2010b). Pour le 
second tri, l'aspect visuel de la pochette (Cunningham et al., 2003; Laplante, 2010b) demeure important pour plusieurs. Pour les plus connaisseurs, les noms des contributeurs ou de la maison de disque sont également utiles (Laplante, 2010b). Les enquêtes à grande échelle révèlent, elles aussi, un besoin pour des métadonnées enrichies. En plus des traditionnelles données bibliographiques, les gens rapportent utiliser l'image de la pochette des albums, des informations sur le genre musical auquel un artiste appartient, de même que des informations générales sur l'artiste, ses influences et sa musique lors de leurs recherches de musique (Lee et al., 2016; Lee et Downie, 2004).

Bien que le domaine soit en émergence, il est néanmoins possible d'observer l'évolution dans les habitudes d'écoute de musique et de recherche d'information musicale. Jin Ha Lee, qui avait réalisé en collaboration avec J. Stephen Downie une enquête sur le sujet en 2004, a repris en 2012, avec de nouveaux collaborateurs, la même enquête qu'elle a mise à jour et enrichie afin de pouvoir en comparer les résultats (Lee et al., 2016; Lee et Waterman, 2012). Cette comparaison fait ressortir les changements qui ont eu lieu dans les pratiques informationnelles des gens au cours des dernières années. Sans surprise, les services d'écoute de musique en ligne avaient grandement gagné en popularité. Alors que 77,6 \% des répondants du sondage de 2004 disaient écouter au moins occasionnellement de la musique par l'intermédiaire de radios Web ou de services d'écoute de musique en continu, c'est la presque totalité des répondants, soit 96,6 \%, de l'enquête de 2012 qui le faisait. Pour près du tiers des répondants (33,2\%), il s'agissait même d'une activité quotidienne (Lee et al., 2016). Les résultats montrent par ailleurs que les services de partage de vidéos tels que YouTube sont de plus en plus utilisés pour écouter et découvrir de la musique (Lee et al., 2016). Les réponses aux questions ouvertes du sondage de 2012 montrent que les jeunes adultes apprécient les services de musique en continu et les sites tels que YouTube en raison de leur offre presque illimitée et parce que ces services les exposent à de nouvelles musiques, leur permettant ainsi d'y faire de véritables découvertes (Lee et Waterman, 2012). Les auteures de l'enquête suggèrent également d'autres raisons pour expliquer la popularité de ces services. D'abord, l'espace de stockage limité des téléphones mobiles et des tablettes rend les systèmes infonuagiques plus attrayants pour les usagers. De la même façon, gérer une collection de fichiers importante constitue un véritable défi étant donné que les gens possèdent bien souvent plus d'un appareil (téléphone, ordinateur, tablette) et qu'ils changent ces appareils régulièrement. Enfin, ces services permettent aux utilisateurs d'écouter de la musique qu'ils apprécient en ce moment, mais qu'ils ne désirent pas nécessairement acheter (Lee et Waterman, 2012). Des différences dans les résultats de 2004 et 2012 sont également notées dans les habitudes d'achat des répondants. Alors que la proportion des répondants qui rapportaient acheter des fichiers musicaux en ligne avait augmenté de façon importante (de $37,7 \%$ à 83,1\%), la proportion de ceux qui achètent des enregistrements musicaux sur support physique avait décru de $10 \%$, passant de $81,2 \%$ à $71,2 \%$.

II est par ailleurs intéressant de noter que les répondants de ces deux enquêtes provenaient pour la majorité des États-Unis (73,8 \% pour l'enquête de 2004 et 60,3\% 
pour celle de 2012). Or, une enquête réalisée auprès d'étudiants d'universités hongkongaises montre qu'il existe des différences entre les pays (Hu et al., 2014). D'une part, les services disponibles ne sont pas les mêmes. Ainsi, le deuxième site le plus fréquenté pour la musique par les étudiants de Hong Kong était KKBOX, un service d'écoute de musique en continu basé à Taïwan et disponible dans certains pays asiatiques uniquement. D'autre part, les habitudes d'écoute de la musique diffèrent. Par exemple, Hu et ses collaboratrices notent que les étudiants hongkongais se fient davantage à la popularité et beaucoup moins aux genres musicaux dans leurs recherches (Hu et al., 2014).

Le fait que les pratiques de recherche de musique des gens aient changé de façon assez importante en aussi peu que huit ans et que des enquêtes similaires réalisées au même moment aux États-Unis et à Hong Kong produisent des résultats différents démontrent qu'il est important de répliquer les études régulièrement et auprès d'autres populations.

\section{Objectif de l'étude}

Cette étude tente de mieux connaître les habitudes de consommation de musique et le comportement dans la recherche d'information musicale des jeunes adultes québécois.

Plus précisément, cette étude vise à répondre aux questions de recherche suivantes :

- Quel(s) appareil(s) les jeunes adultes utilisent-ils pour écouter de la musique?

- Comment acquièrent-ils des enregistrements musicaux?

- Quels services ou sources utilisent-ils pour découvrir de la musique?

- Quels services en ligne utilisent-ils pour écouter de la musique?

En outre, cette étude permet de comparer le comportement dans la recherche d'information des jeunes adultes québécois à celui des jeunes adultes américains car, tout comme Lee et ses collaboratrices, plusieurs des questions de cette enquête ont été puisées (et adaptées) de l'étude de Lee et Downie (2004).

\section{Méthodologie}

Un questionnaire en ligne, inspiré du questionnaire de Lee et Downie (2004), a été conçu pour cette étude. II comprend 20 questions couvrant deux axes thématiques - les habitudes de recherche d'information musicale (six questions) et les habitudes d'écoute de musique (dix questions) - auxquelles s'ajoutent des questions démographiques (quatre questions). Le questionnaire était composé de questions ouvertes et fermées et utilisait principalement l'échelle de Likert et des choix multiples. Remplir le questionnaire prenait de cinq à dix minutes et, à la fin, les répondants pouvaient laisser leur adresse courriel pour participer à un tirage afin de gagner une carte-cadeau de $25 \$$ chez Amazon. Les participants ont été informés que les données resteraient confidentielles et qu'ils pouvaient abandonner le questionnaire à tout moment. Notons à ce sujet que puisque cette étude faisait appel à des sujets humains, elle a fait l'objet 
d'une évaluation par le Comité d'éthique de la recherche en arts et sciences de l'Université de Montréal, qui a accordé un certificat d'éthique.

Le questionnaire a été conçu au moyen du service de sondage en ligne SurveyGizmo. Un prétest fut réalisé auprès de quelques utilisateurs avant sa mise en ligne le 5 mars 2014. La période d'ouverture était de 21 jours. Le recrutement s'est fait par l'intermédiaire des médias sociaux, plus précisément de Facebook et de Twitter. Certaines communautés susceptibles de regrouper des amateurs de musique ont été ciblées, notamment des groupes d'étudiants en musique ou en musicologie ainsi que des étudiants en sciences de l'information. Les auteurs ont également diffusé l'invitation au moyen de leurs comptes personnels sur ces deux plateformes et les gens qui ont reçu l'invitation étaient évidemment libres de rediffuser l'annonce de recrutement dans leur propre réseau. II s'agit donc d'un échantillon de convenance, non probabiliste. Notons à cet égard que les autres enquêtes sur le sujet ont adopté une méthode d'échantillonnage similaire : Hu, Lee et Wong (2014) ont recruté leurs participants au moyen de Facebook et Lee et ses collègues (Lee et al., 2016; Lee et Waterman, 2012) ont recruté une part de leurs participants au moyen de Facebook, de Twitter et de Google+.

Pour participer à l'enquête, il fallait être âgé entre 18 et 35 ans et habiter au Québec. 278 questionnaires complétés et valides ont été reçus. Certaines questions étant facultatives, le nombre de réponses par question varie légèrement. $38 \%$ des répondants sont des hommes et $62 \%$, des femmes. L'âge moyen était de 28 ans et l'âge médian, de 24 ans. Au moment de l'enquête, 53,3\% étaient des travailleurs, $44,6 \%$ étaient des étudiants et 2,2\% étaient sans emploi. Enfin, la majorité avait un niveau de scolarité élevé : 76,6 \% détenaient un diplôme universitaire.

Ce portrait de notre échantillon porte les traces du biais induit par la méthode d'échantillonnage retenue. Le niveau de scolarité y est plus élevé que dans la population québécoise en général. En 2014, 34,4\% des 25 à 34 ans possédaient un diplôme universitaire (Institut de la statistique du Québec, 2015). Le haut niveau de scolarité des répondants est également lié à l'inégalité de la distribution hommesfemmes de notre échantillon puisque les femmes sont plus nombreuses à fréquenter l'université que les hommes (34 \% contre $25 \%$ chez les 20-24 ans en 2012) (Gauthier, 2014).

Selon une enquête de Statistique Canada, plus une personne est scolarisée, plus elle est susceptible de consommer des biens culturels liés à la musique (Ewoudou, 2005). Par exemple, en 2005, 10,1 \% des Canadiens qui n'avaient pas de diplôme d'études secondaires assistaient occasionnellement à des spectacles de musique populaire, contre $24,4 \%$ de ceux qui détenaient un diplôme universitaire. De manière similaire, 40,2 \% des Canadiens qui n'avaient pas de diplôme d'études secondaires écoutaient fréquemment de la musique sur disques compacts, cassettes ou disques vinyle, contre 48,7 \% de ceux qui détenaient un diplôme universitaire (Ewoudou, 2005). II est donc probable que les répondants à notre enquête écoutaient et acquéraient davantage de musique que la moyenne. Par ailleurs, notons que cette limite comporte 
aussi des avantages : la majorité des enquêtes réalisées sur le même sujet comptaient également parmi leurs répondants une grande proportion de personnes étudiant à l'université ou possédant un diplôme universitaire, que ce soit parce qu'il s'agissait de leur population cible ou en raison du mode de recrutement utilisé (Hu et al., 2014; Lee et al., 2016; Lee et Downie, 2004; Lee et Waterman, 2012; Tepper et Hargittai, 2009). Cette similitude facilite la comparaison des résultats.

\section{Résultats et discussion}

\section{Habitudes d'écoute et de consommation de la musique}

Nous avons posé quelques questions à nos participants afin de dresser un portrait de leurs habitudes de consommation de musique. Plus de quatre répondants sur cinq ont dit écouter de la musique sur une base quotidienne : de une à deux heures par jour pour $44,4 \%$ et plus de deux heures par jour pour 36,6 \% des répondants. 83,7 \% des répondants ont rapporté s'être déplacés dans la dernière année pour assister à un concert musical. De ce nombre, la plupart avaient assisté à ce type d'activité entre deux et cinq fois dans l'année (49,8\%). Enfin, nous leur avons demandé s'ils savaient jouer d'un instrument de musique. $31,8 \%$ on dit savoir jouer d'un instrument en tant qu'amateur et $15,5 \%$, en tant que professionnel, pour un total de $47,3 \%$.

À la question « Parmi les formats suivants, lesquels utilisez-vous régulièrement pour écouter de la musique? », les MP3 et autres formats de fichiers compressés arrivent en première place $(88,8 \%)$. La deuxième place va à l'écoute de musique en ligne (services d'écoute de musique en continu) (78\%). Puis, en troisième et quatrième places, nous retrouvons deux médiums traditionnels, soit les CD $(61,7 \%)$ et la radio FM $(57 \%)$. L'ensemble des résultats est présenté à la Figure 1.

Le pourcentage d'utilisateurs des services d'écoute de musique en continu, bien qu'élevé, est bien en deçà de ce que Lee et ses collaboratrices observent ${ }^{1}$. En effet, ce sont $94,9 \%$ de leurs répondants qui ont rapporté utiliser ces services (Lee et Waterman, 2012). Cette différence pourrait s'expliquer par le fait que l'offre était nettement plus limitée au Canada au moment du sondage comparativement à ce que l'on trouvait déjà en 2012 aux États-Unis. Cette différence dans l'offre s'explique par le fait que les fournisseurs de services doivent conclure des ententes avec les détenteurs de droits (principalement les maisons de disques) pour pouvoir diffuser leur musique, et ces ententes varient d'un pays à l'autre. Les données de l'International Federation of the Phonographic Industry (IFPI) confirment que les services d'écoute de musique en continu sont effectivement moins populaires au Canada que dans les autres pays

\footnotetext{
${ }^{1}$ Nos résultats restent cependant bien au-dessus de ceux obtenus par Kamalzadeh, Baur et Möller (2012). Cette enquête canadienne, antérieure de quelques années à la nôtre (la période de collecte des données n'est pas précisée), montre que seuls 33,8\% des répondants utilisaient des services de musique en ligne. II faut cependant noter que non seulement moins de services étaient alors disponibles au Canada, mais les répondants affichaient un profil assez différent des nôtres. La très grande majorité des 222 répondants de cette enquête étaient des étudiants et des professeurs en informatique et en génie de la Simon Fraser University.
} 
développés, notamment les États-Unis : alors que $14 \%$ des revenus liés à la musique numérique provenaient d'abonnements à des services musicaux en ligne en 2014, cette proportion n'était que de $8 \%$ au Canada. En contrepartie, la vente de fichiers musicaux au Canada représentait $88 \%$ des revenus, contre $55 \%$ aux États-Unis (IFPI, 2015).

Mais dans notre sondage comme dans celui de Lee et ses collaboratrices, la proportion d'utilisateurs de ces services est très élevée, surtout si on considère que ces services sont très récents. Les services d'écoute de musique en continu sont en effet en pleine croissance. Toujours selon l'IFPI, à l'échelle mondiale, les revenus provenant de ce secteur auraient grimpé de $39 \%$ en 2014 et représenteraient $23 \%$ de tous les revenus liés à la musique numérique. On estimait à 41 millions le nombre de personnes dans le monde qui payaient pour un abonnement à un service d'écoute de musique en continu en 2014, comparativement à 28 millions en 2013 et à 8 millions en 2010 (IFPI, 2015).

Le fait que les fichiers musicaux compressés et les services d'écoute de musique en continu représentent tous deux une très forte majorité d'utilisateurs chez nos répondants indique que plusieurs utilisent les deux simultanément. Ce constat est surprenant étant donné que plusieurs des services musicaux en ligne proposent une offre musicale extrêmement étendue. Cela pourrait s'expliquer de différentes façons. II est possible que les efforts et le temps alloués à bâtir une collection numérique - à transférer des CD, à ajouter des métadonnées ou à préparer soigneusement des listes d'écoute - incitent les gens à continuer à exploiter leur collection musicale personnelle. De plus, malgré l'offre étendue des services d'écoute de musique en continu, celle-ci n'est pas exhaustive. Enfin, bien que certains services musicaux en ligne permettent de télécharger un certain nombre de fichiers, un abonnement payant est habituellement nécessaire. II est donc possible que les gens utilisent les fichiers compressés dans leur collection lorsqu'ils n'ont pas accès à Internet ou que l'accès est limité, par exemple lorsque leur forfait de téléphonie mobile limite la quantité de données pouvant être téléchargées. 


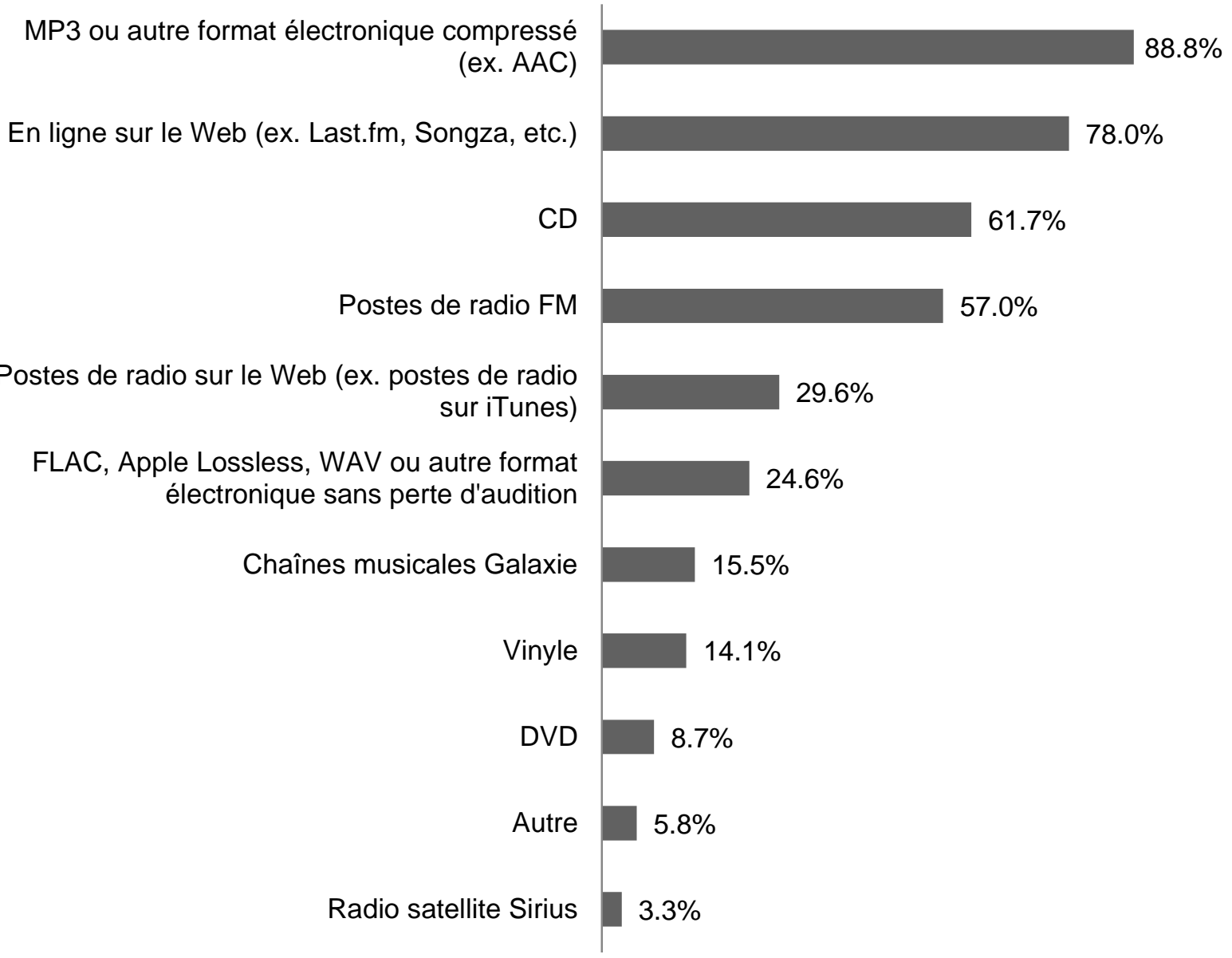

Figure 1. Réponses à la question $3:$ :Parmi les formats suivants, lesquels utilisez-vous régulièrement pour écouter de la musique? » $(n=277)$.

Nous avons également cherché à savoir quels étaient les dispositifs que les répondants utilisaient pour écouter de la musique. Nous pouvons observer à la Figure 2 qu'une très forte majorité des répondants écoutaient régulièrement de la musique sur un ordinateur $(89,5 \%)$. Ce dispositif devance largement les appareils portatifs comme les lecteurs MP3 $(59,2 \%)$ et les téléphones mobiles (43,7\%). On constate par ailleurs que la chaîne stéréophonique classique arrive en troisième place $(53,4 \%)$ et demeure donc populaire auprès des jeunes adultes interrogés.

Ces résultats semblent contredire ceux obtenus par Krause et North (2016) qui ont interrogé 342 personnes de 16 à 72 ans du Royaume-Uni et des États-Unis sur les appareils utilisés pour écouter de la musique. Leur étude montre que les appareils mobiles arrivent en tête (33,8\%), suivis de près par l'ordinateur (32,6\%). Il faut cependant noter que la question était formulée différemment : on demandait aux participants quel était leur appareil de lecture préféré. II est donc possible que l'on utilise l'ordinateur surtout pour des raisons de commodité, mais que l'on apprécie davantage les appareils mobiles. De plus, les choix de réponses n'étaient pas exactement les mêmes; l'ordinateur et Internet ont été considérés comme deux 
réponses distinctes dans l'étude de Krause et North. On confond ainsi appareil de lecture d'un côté et support (p. ex. : fichiers numériques, disques compacts) ou mode de lecture de l'autre (lecture en mode local ou en continu). Il est donc impossible de savoir si les gens préfèrent accéder aux services musicaux sur Internet au moyen de leur ordinateur ou de leur appareil mobile.

Il peut paraître étonnant de voir l'ordinateur devancer les appareils portatifs. La popularité de l'ordinateur comme dispositif d'écoute pour la musique peut s'expliquer de diverses façons. D'abord, il s'agit d'un appareil flexible, qui permet d'écouter la musique sur différents formats et provenant de différentes sources : CD, DVD, fichiers musicaux (compressés ou non), lecture de musique ou de vidéos en ligne, etc. De plus, comparativement aux appareils mobiles, l'ordinateur permet plus facilement l'écoute de musique à plusieurs et dispose d'un espace de stockage nettement plus important. Enfin, le coût élevé des forfaits de données mobiles au Canada (Nordicity Group Limited, 2016) pourrait également expliquer l'utilisation limitée des appareils mobiles pour écouter de la musique si cette musique provient d'un service d'écoute en continu.

Mais peut-être encore plus surprenant est le fait que la majorité des répondants utilisaient encore une chaîne stéréophonique. II est possible, dans ce cas, que l'on continue d'utiliser ce dispositif par nostalgie ou pour écouter des enregistrements musicaux qui ne sont pas disponibles en ligne et qui n'ont pas été transférés sur un ordinateur. Les habitudes sont parfois aussi fortes. Par exemple, tel que les résultats de la question précédente le démontrent, même les services de musique en ligne n'ont pas réussi à éradiquer les chaînes musicales Galaxie (aujourd'hui Stingray Musique) disponibles via les opérateurs de télévision.

Enfin, les résultats des questions 3 et 4 suggèrent que plusieurs de nos répondants écoutaient de la musique sur une variété de supports et au moyen de divers dispositifs.

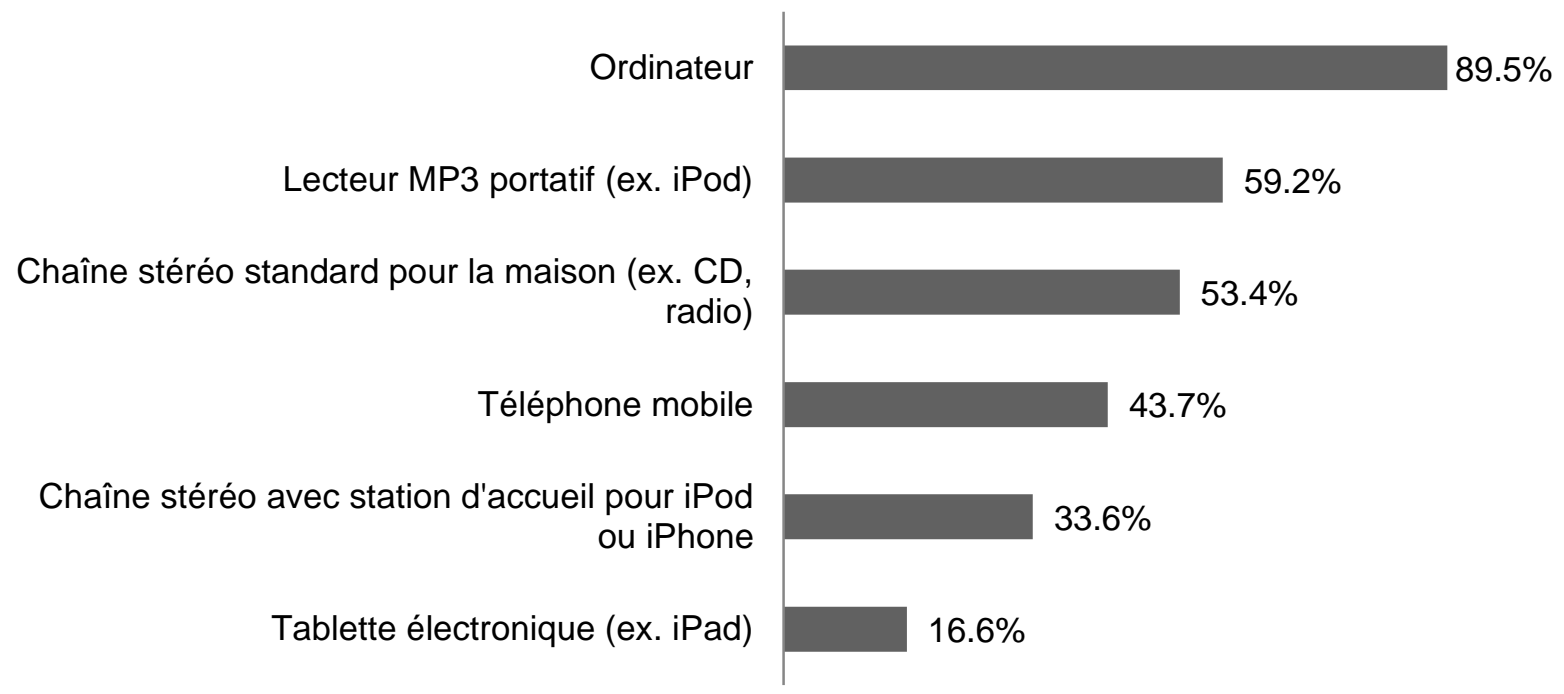

Figure 2. Réponses à la question 4 : «Parmi ces dispositifs, lesquels utilisez-vous régulièrement pour écouter de la musique? » $(n=277)$. 
Les répondants étaient également invités à indiquer les formats dans lesquels ils acquéraient de la musique (voir Figure 3). Les formats musicaux numériques compressés étaient de loin les plus populaires : $80,1 \%$ des répondants avaient fait l'acquisition de musique dans ce format au cours des 12 derniers mois. Le CD (58,6\%) et même le disque vinyle (14,6\%) demeurent néanmoins populaires. Cependant, nos données ne nous permettent pas de savoir si l'achat de musique sur ces supports traditionnels est courant ou occasionnel.

La popularité des disques vinyle et des CD pour l'acquisition de musique peut paraître surprenante étant donné le déclin important qu'ont connu ces supports. En effet, les études indiquent que les ventes de musique sur support physique avaient baissé au Québec de 7,1\% entre 2013 et 2014 (Fortier, 2015) et que les revenus liés à la vente de ces supports avaient baissé de 11,7\% à l'échelle internationale de 2012 à 2013 (Smirke, 2014). Par contre, malgré le déclin des ventes de musique sur support physique, il est pertinent de rappeler que, selon l'IFPI, la vente de ces produits rapportait autant que les revenus associés à la musique numérique (par la vente, la publicité et les abonnements) en 2015. Chacun de ces secteurs représentait en effet $46 \%$ des revenus de l'industrie de la musique (IFPI, 2015). En 2015, la vente de disques vinyle était d'ailleurs en croissance de $30 \%$ par rapport à 2014 au Canada selon le rapport de Nielsen (2016). La vente de musique sur support physique n'est donc pas encore chose du passé.

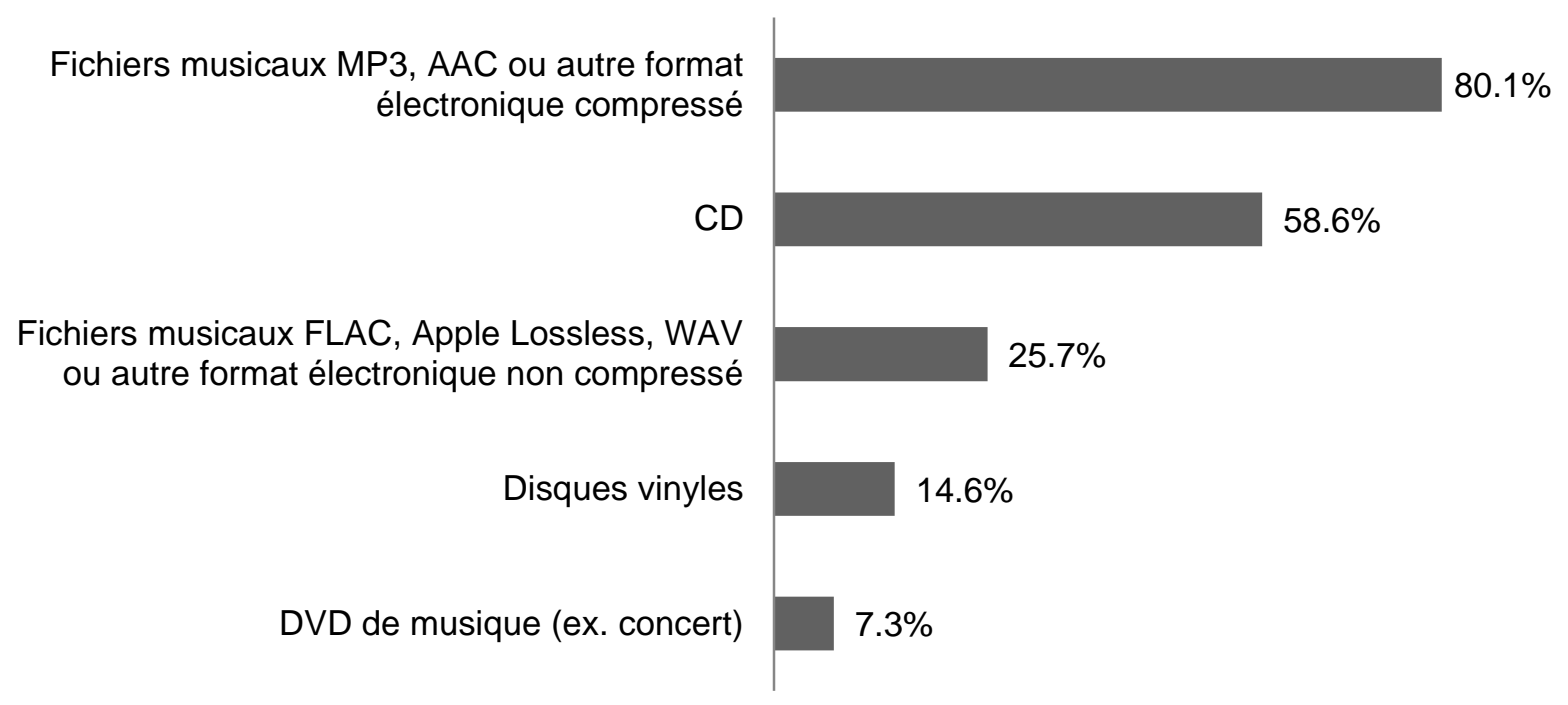

Figure 3. Réponses à la question 5 : «Depuis les douze derniers mois, avez-vous fait l'acquisition d'un ou de plusieurs ... » $(n=261)$.

Pour acquérir des fichiers musicaux numériques, $71,7 \%$ des répondants ont dit utiliser un service payant (voir Figure 4) et 57,5\% se servaient des systèmes pair-à-pair (voir Figure 5). Le téléchargement de fichiers musicaux numériques, qu'il soit légal ou non, était donc une activité très populaire auprès des jeunes interrogés. Ces résultats sont par contre inférieurs à ceux obtenus par Lee, Cho et Kim (2016) : 81,9\% de leurs répondants ont en effet rapporté télécharger de la musique disponible gratuitement et 
$83,1 \%$ ont rapporté acheter de la musique en format numérique. Ils ont d'ailleurs pu constater que, parmi les activités en ligne reliées à la musique, l'achat et le téléchargement de musique étaient l'activité ayant connu la plus forte hausse en popularité chez les répondants (+45,4\%) entre le sondage effectué en 2004 et celui effectué en 2012.

L'examen des réponses de nos répondants permet en outre de constater que plusieurs utilisateurs des services payants téléchargent également de la musique de façon illégale.

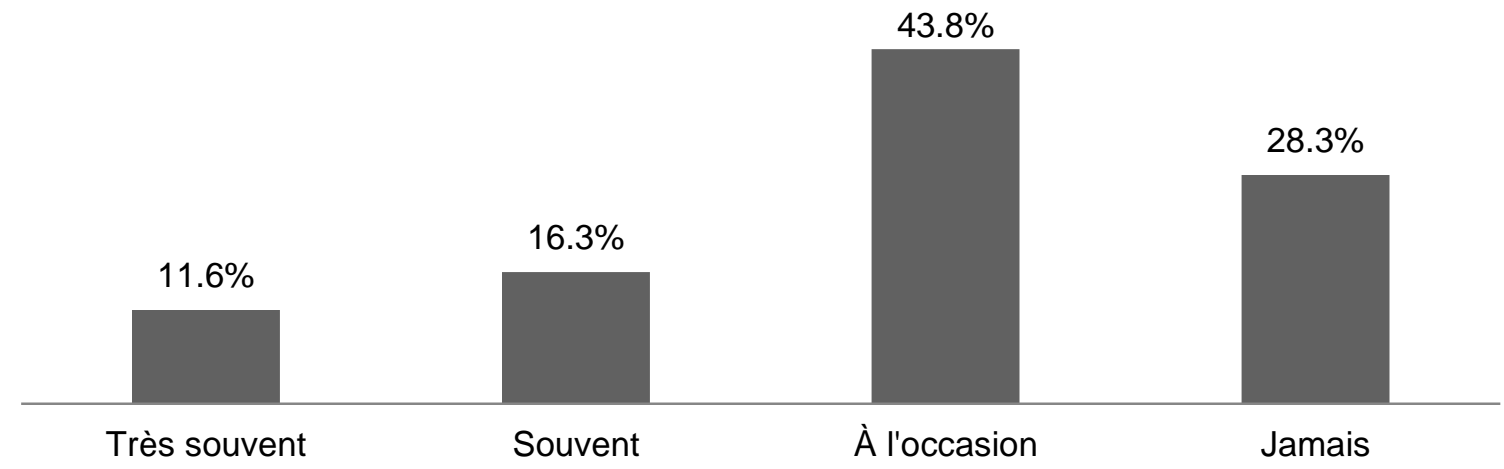

Figure 4. Réponses à la question 10 : «Vous arrive-t-il de télécharger de la musique sur un service payant comme iTunes ou archambault.ca? » $(n=276)$.

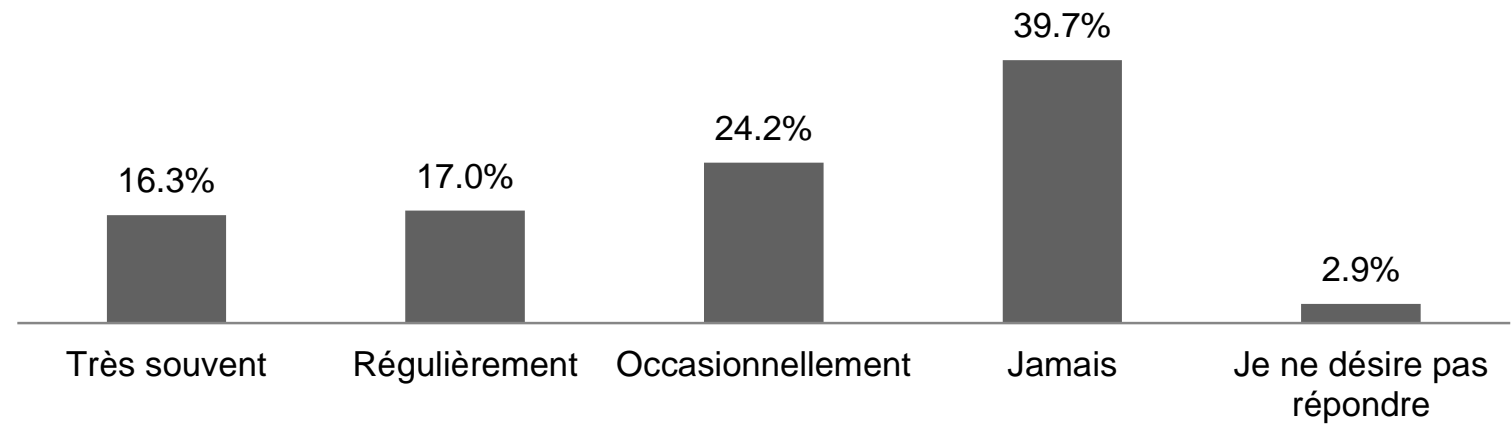

Figure 5. Réponses à la question 11 : «Vous arrive-t-il de télécharger de la musique via un système peer-to-peer (ex. BitTorrent, Pirate Bay, etc.) ? » $(n=277)$.

Les participants ont également été appelés à dire comment leurs habitudes d'achat de musique avaient évolué au cours des deux dernières années. Plus de la moitié des répondants $(53,7 \%)$ ont rapporté acheter plus ou autant de musique qu'il y a deux ans, alors qu'un peu plus du tiers (37,6 \%) ont estimé en acheter moins (voir Figure 6). Ces résultats semblent contredire les données de l'Observatoire sur la culture et les communications du Québec, qui indiquent une baisse de 14,9\% des ventes d'enregistrements audio (en numérique et sur support physique) au Québec entre 2012 et 2014 (Fortier, 2015). Cependant, il paraît important de rappeler que les répondants sont de jeunes adultes. II est possible que plusieurs soient entrés récemment sur le marché du travail, améliorant ainsi leur situation financière et, par le fait même, leur pouvoir d'achat. 


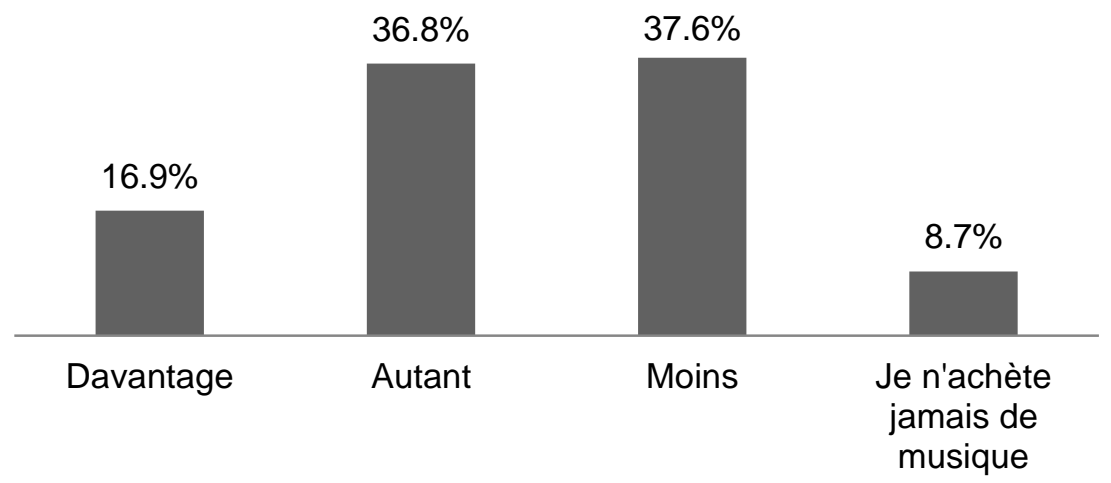

Figure 6. Réponses à la question 6 : «Achetez-vous autant de musique qu'il y a deux ans? J'en achète... » $(n=277)$.

Les participants étaient ensuite appelés à donner leur opinion sur l'offre de musique gratuite et légale sur le Web (par exemple la musique pouvant être écoutée sur les radios Web ou les sites des artistes). Près des deux tiers (63,5\%) des participants se sont dits satisfaits ou très satisfaits de cette offre, $17,2 \%$ n'avaient pas d'opinion et $15,7 \%$ étaient peu ou pas satisfaits (voir Figure 7 ).

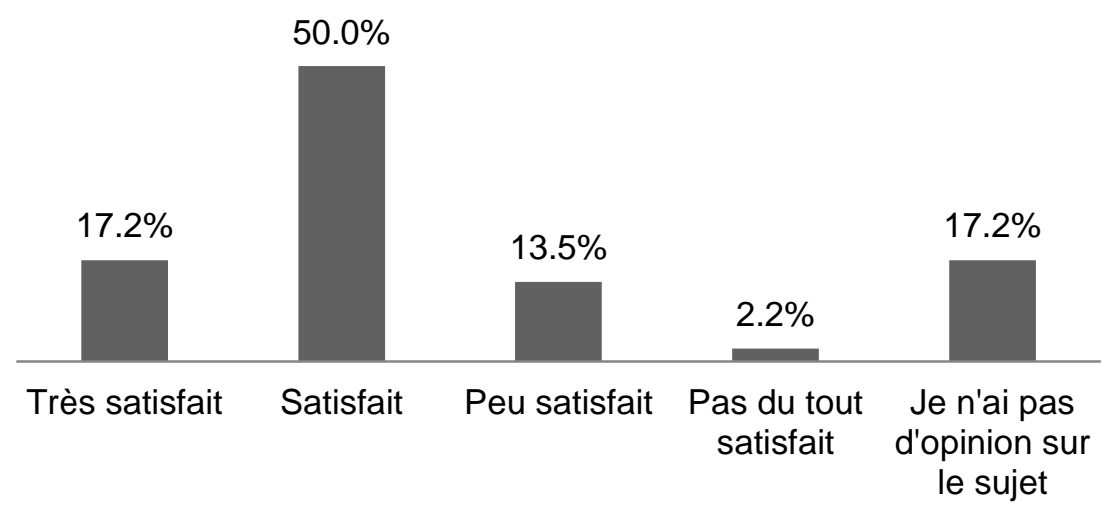

Figure 7. Réponses à la question 13 : « De façon générale, êtes-vous satisfait de l'offre musicale disponible gratuitement et légalement sur le Web ? » $(n=274)$.

\section{La découverte de nouvelles musiques}

Nous avons ensuite demandé aux répondants d'indiquer dans une liste les sites Web ou autres sources d'information qu'ils utilisaient pour découvrir de la nouvelle musique et d'indiquer à quelle fréquence ils les utilisaient. Le Tableau 1 et la Figure 8 présentent le pourcentage de répondants utilisant chaque type de source. 


\section{Tableau 1}

\section{Sites Web et endroits utilisés pour découvrir de la musique}

\begin{tabular}{lr}
\hline \hline Site ou endroit & Pourcentage \\
\hline \hline Plateformes de diffusion de vidéos (ex. YouTube, Vimeo, etc.) & $94,4 \%$ \\
\hline Collection virtuelle d'une personne de mon entourage (ex. fichiers MP3) & $73,9 \%$ \\
\hline Collection physique d'une personne de mon entourage (ex. CD, vinyles, etc.) & $72,7 \%$ \\
Services de recommandations musicales et d'écoute de musique en ligne (ex. & $67,1 \%$ \\
Grooveshark, Last.fm, Songza) & $61,8 \%$ \\
Journaux et magazines généralistes (ex. La Presse, Voir) & $60,8 \%$ \\
Google & $55,5 \%$ \\
Blogues et sites Web personnels dédiés à la musique & $54,1 \%$ \\
Plateformes de diffusion d'enregistrements sonores (ex. SoundCloud) & $51,6 \%$ \\
Disquaire & $46,5 \%$ \\
Wikipédia & $36,2 \%$ \\
Journaux et magazines spécialisés (ex. Les Inrockuptibles, Billboard, Rolling Stone) & $26,1 \%$ \\
Bibliothèque publique ou universitaire & $20,8 \%$ \\
Guides musicaux (ex. AllMusic) &
\end{tabular}




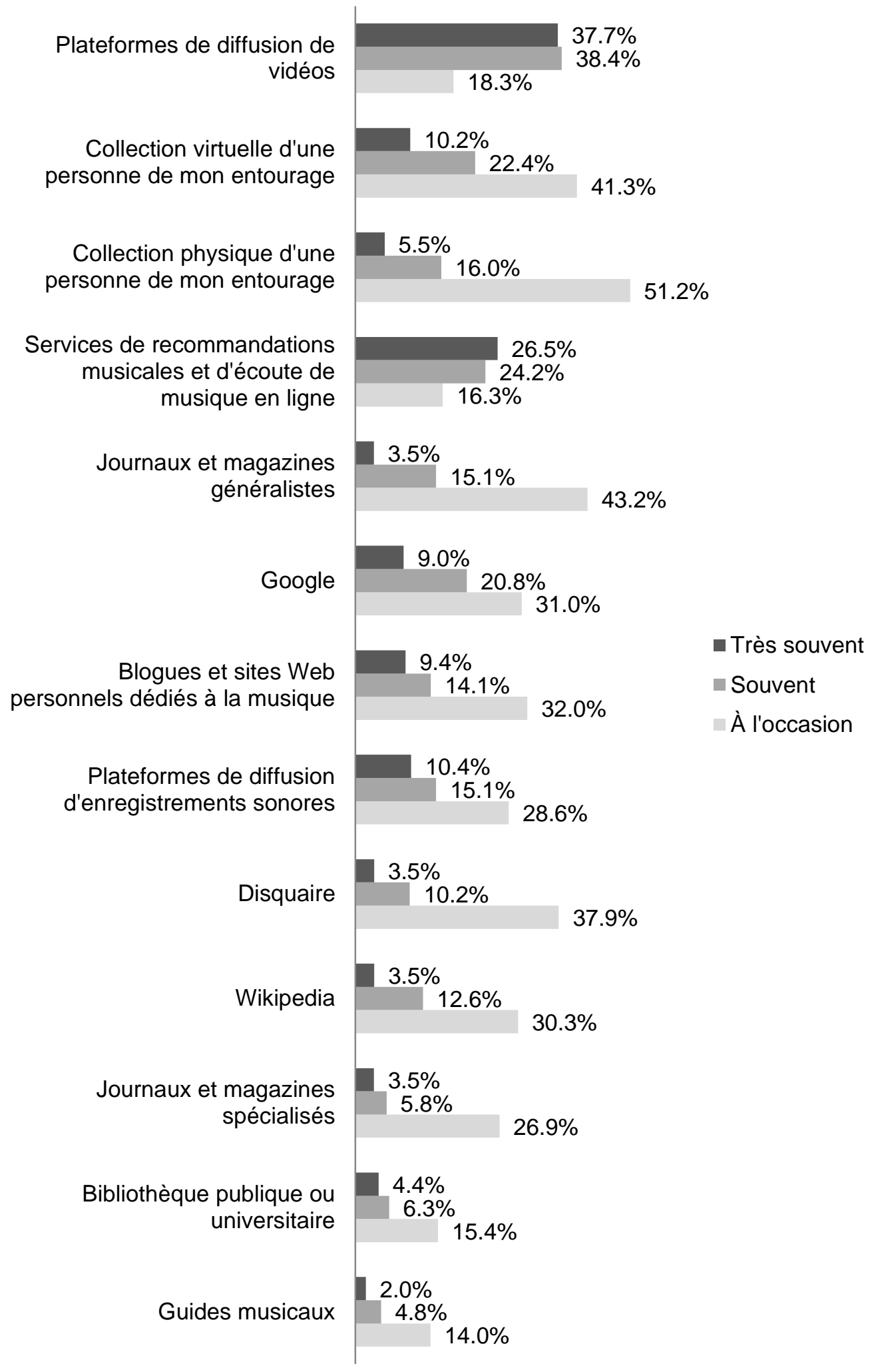

Figure 8. Fréquence d'utilisation des sites Web et endroits utilisés pour découvrir de la musique. 
Les plateformes de diffusion de vidéos telles que YouTube arrivent clairement en première place avec $94,4 \%$ d'utilisateurs parmi les répondants. C'est également le type de source que les répondants utilisaient le plus fréquemment, car ces plateformes arrivent également en tête de liste des sources que les répondants ont dit utiliser très souvent $(37,7 \%)$ ou souvent $(38,4 \%)$. Les services de recommandations musicales et d'écoute de musique en continu ne figurent qu'en quatrième position avec $67,1 \%$ de répondants qui disent utiliser ces services pour découvrir de la musique. Notons cependant que ceux qui utilisent ces services les utilisent fréquemment puisque $26,5 \%$ ont indiqué les utiliser très souvent et $24,2 \%$, souvent. Au contraire, bien qu'une grande majorité de répondants ont dit consulter les collections de disques de personnes de leur entourage $(72,7 \%)$ ou consulter des journaux et des magazines généralistes $(61,8 \%)$ dans le but de découvrir de la musique, ces sources étaient utilisées beaucoup plus occasionnellement que les autres sources en tête de liste.

La fréquence d'utilisation n'est cependant pas le seul élément dont il faut tenir compte. Nous souhaitions également savoir quelle est l'efficacité de ces sources selon nos répondants. Ceux-ci ont ainsi été invités à classer par ordre d'importance les sources par lesquelles ils découvrent de la musique (Tableau 2). La liste comprenait sept sources. La compilation des résultats s'est faite en attribuant un pointage de sept à une ressource placée en première position et un pointage de un à une source placée en septième position. Comme 250 répondants ont répondu à cette question, le score maximal était de 1750. Avec un score de 1459, les suggestions de l'entourage (amis, collègues ou famille) arrivent en première place, suivies des recommandations des services de musique en continu tels que Last.fm ou Songza avec un score de 1171. Les suggestions des disquaires arrivent en dernière place avec un score très faible de 368 .

Ces résultats sont globalement conformes aux résultats des autres études réalisées sur le sujet. De fait, les enquêtes de Lee et ses collaborateurs ont également démontré que c'est d'abord vers leurs amis et les membres de leur famille que les gens se tournent pour obtenir de l'aide pour trouver de la musique, dans une proportion de $80,9 \%$ en 2004 (Lee et Downie, 2004) et de 82,8 \% en 2012 (Lee et al., 2016). Par ailleurs, la faible popularité des disquaires et plus globalement des spécialistes avait également été observée dans d'autres études (Cunningham et al., 2003; Laplante, 2010b; Lee et al., 2016; Lee et Downie, 2004).

Les études qualitatives nous expliquent à la fois l'importance des membres de l'entourage et l'impopularité des disquaires et des bibliothécaires. Selon Cunningham, Reeves et Britland (2003), les gens seraient intimidés d'aller consulter le personnel des magasins de disques, car ils craignent d'être perçus comme ayant peu de culture musicale. Consulter ces spécialistes représente donc pour plusieurs une solution de dernier recours. Par ailleurs, selon Laplante et Downie (2006), les gens considèrent que les goûts musicaux sont subjectifs et personnels et qu'il est donc difficile de déterminer si on peut faire confiance à une personne qu'on ne connaît pas, comme un disquaire. Au contraire, les membres de notre entourage peuvent fournir des recommandations personnalisées, car ils connaissent nos goûts. Nous pouvons également mieux évaluer la pertinence de leurs recommandations puisque nous connaissons aussi les leurs. 


\section{Tableau 2}

Réponses à la question 9 : "Par qui ou par quoi découvrez-vous le plus souvent de nouvelles musiques ou de nouveaux artistes musicaux? " $(n=250)$

\begin{tabular}{clr}
\hline \hline Rang & Réponse & Score obtenu sur 1750 \\
\hline \hline 1 & Suggestions de l'entourage (amis, collègues, famille) & 1459 \\
2 & Suggestions fournies par des services musicaux en ligne & 1171 \\
& (Songza, Last.fm, YouTube, etc.) & 941 \\
3 & Musique entendue à la radio & 902 \\
4 & Musique entendue à la télévision ou au cinéma & 844 \\
5 & Musique entendue dans un espace public (bar, café, restaurant, & \\
& etc.) & 820 \\
7 & Critiques musicales (journaux, radio, télévision, etc.) & 368
\end{tabular}

L'importance des services d'écoute de musique en continu comme source de découvertes musicales est un phénomène relativement nouveau, observé également par Lee et ses collaboratrices. Dans l'enquête de 2012, 50,8 \% de leurs répondants ont indiqué utiliser les fonctions de recommandations de ces services (Lee et Waterman, 2012 ) et $82,2 \%$ ont dit que le fait d'écouter de la musique au moyen d'un service tel que Pandora ou Spotify les amenait à faire des recherches sur la musique (Lee et al., 2016).

Étant donné la popularité croissante des services d'écoute de musique en continu, nous avons présenté une liste des principaux services disponibles au Canada au moment du sondage et avons demandé aux répondants d'indiquer dans chaque cas s'ils connaissaient le service et s'ils l'utilisaient (voir Figure 8). À noter que nous n'avons inclus dans la figure que les services connus par plus de $10 \%$ des répondants. 


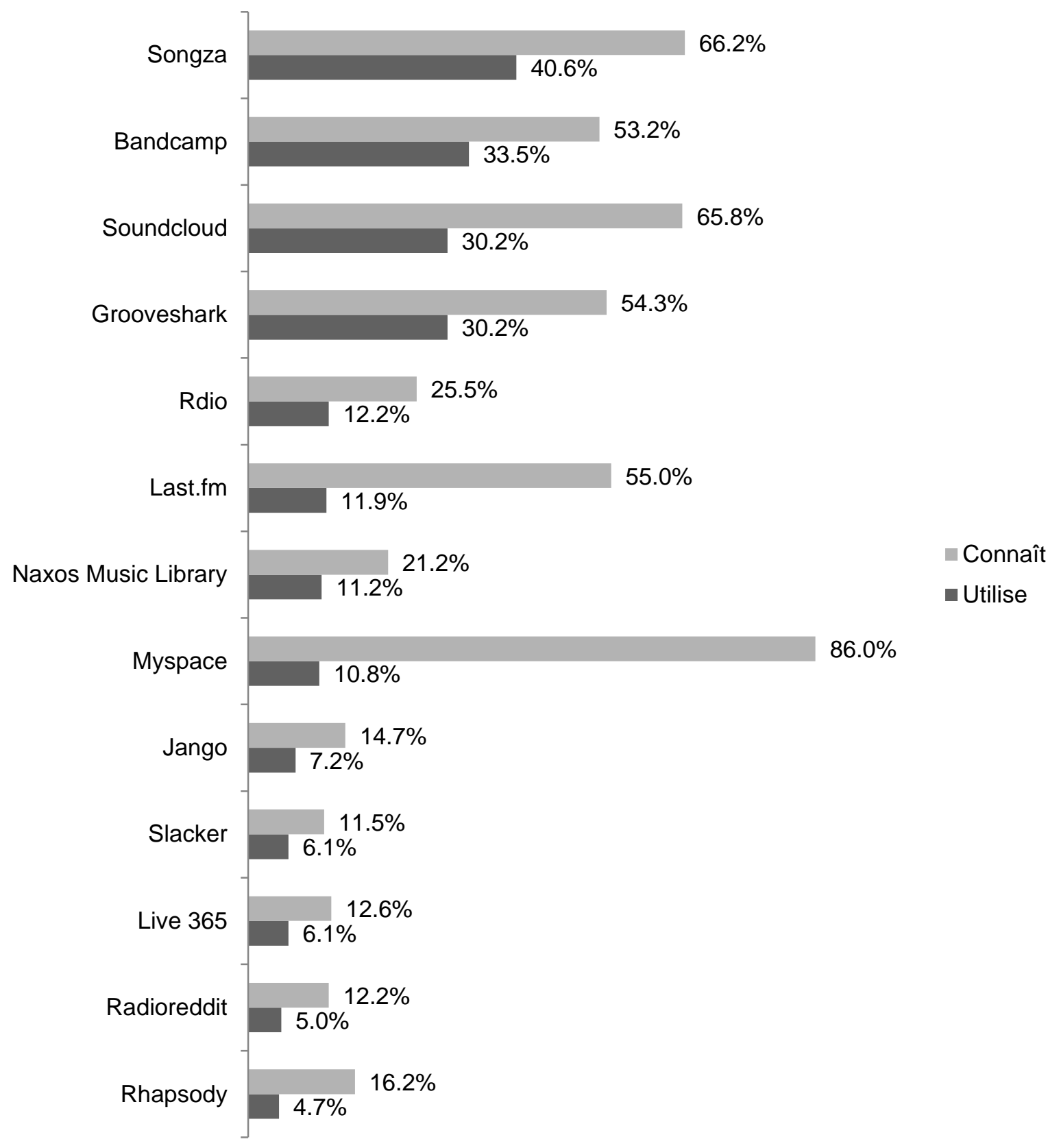

Figure 8. Connaissance et utilisation des services musicaux en ligne, en ordre décroissant d'utilisation.

On constate que le fait qu'un service soit largement connu ne garantit pas son utilisation. De fait, bien que $86 \%$ des participants ont dit connaître Myspace, seuls $10,8 \%$ l'utilisaient réellement. Cet écart, que l'on constate également avec Last.fm, s'explique par l'ancienne notoriété de ces sites. En effet, Myspace et Last.fm sont parmi les premiers services d'écoute de musique en ligne apparus sur le Web (ils sont apparus respectivement en 2003 et 2002). Ils ont connu dans leurs premières années d'existence un grand engouement, mais leur popularité a décliné au fil du temps pour laisser la place à de nouveaux services musicaux. Le deuxième site le plus connu des 
répondants était toutefois aussi le site le plus utilisé. En effet, Songza, que 66,7 \% des répondants ont indiqué connaître, comptait 40,6 \% d'utilisateurs. Dans l'ensemble, on constate qu'aucun de ces services n'est arrivé à rallier la majorité des répondants. Lorsqu'on additionne le pourcentage d'utilisateurs parmi les répondants de l'ensemble des services de la liste, on obtient un nombre bien supérieur à $200 \%$ (alors que, tel que présenté à la Figure 1, $78 \%$ ont dit utiliser ce type de services). Cela nous apprend que plusieurs ne sont pas fidèles à un seul service mais utilisent plutôt plusieurs services simultanément. II serait intéressant d'explorer dans une prochaine étude les raisons pour lesquelles on utilise plus d'un service. Étant donné que les services d'écoute de musique en continu n'offrent pas tous le même catalogue de pièces musicales, on peut penser que les goûts musicaux influencent sans doute le choix de service(s) auquel on a recours et que certains utilisateurs changent possiblement de service en fonction du genre de musique qu'ils souhaitent écouter.

Le portrait de la situation ne serait assurément plus le même aujourd'hui. L'offre est en constante mouvance, si bien que, quelques mois à peine après la collecte des données, la liste des services disponibles est déjà bien différente. Au moment où les participants ont répondu au questionnaire, plusieurs services n'étaient pas encore disponibles au Canada ou n'existaient pas encore, dont Apple Music, Google Play Music, Spotify et Tidal, et d'autres, tels que Rdio et Songza, ont disparu.

Enfin, étant donné qu'aller sur le Web pour découvrir de la musique était une activité très répandue parmi les répondants ( $95 \%$ d'entre eux le faisaient), nous avons voulu savoir s'ils appréciaient cette activité. De ces $95 \%, 87,9 \%$ estimaient qu'il s'agit d'une activité agréable ou très agréable et à peine $2 \%$ n'appréciaient pas l'expérience. Cela rejoint les résultats de l'étude de Laplante et Downie (2011). Des entrevues réalisées avec de jeunes adultes ont révélé que plusieurs considéraient en effet que faire des recherches pour découvrir de la musique à des fins récréatives était une activité plaisante et engageante, certains considérant même qu'il s'agissait d'un loisir en soi.

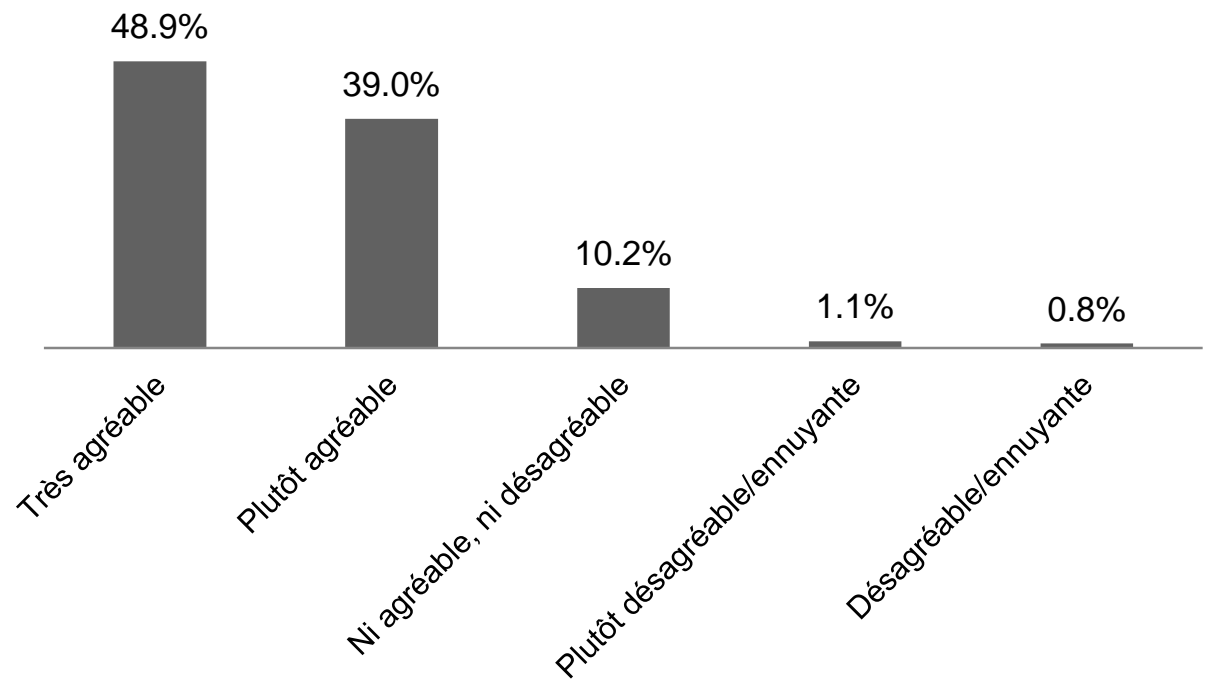

Figure 9. Réponses à la question 7 : «Pour vous, aller sur le Web pour découvrir de nouvelles musiques ou de nouveaux artistes musicaux est une activité ... » $(n=264)$. 


\section{Conclusion}

Les services d'écoute de musique en continu sont résolument en pleine expansion. Non seulement une très forte majorité des répondants les utilisent, mais ils le font très fréquemment. Malgré tout, ces nouveaux services n'ont pas éclipsé les plus anciennes pratiques. Les jeunes adultes interrogés achetaient encore de la musique en format numérique, de même que des $C D$ et des disques vinyle. Même la chaîne stéréophonique classique continue de faire partie de l'univers musical des jeunes Québécois. En fait, plusieurs semblent avoir des pratiques très variées. Ils écoutent de la musique sur divers dispositifs et en différents formats. Et lorsqu'ils écoutent de la musique en ligne, ils utilisent souvent plus d'un service. La musique demeure par ailleurs ancrée dans un contexte social. Malgré le raffinement des algorithmes de recommandation des services musicaux en ligne, les jeunes Québécois continuent d'accorder une grande valeur aux recommandations personnalisées que leur offrent leurs proches et ils apprécient toujours le fait de parcourir les collections musicales physiques ou numériques - de leurs amis, à la recherche de nouvelles musiques. Parmi les services en ligne utilisés pour découvrir de la musique, les plateformes de diffusion de vidéo, telles que YouTube ou Vimeo, surpassent les services d'écoute de musique en continu, ce qui rappelle l'importance de l'aspect visuel dans la recherche d'information musicale. Mais les services d'écoute de musique en ligne prennent aussi progressivement leur place et on peut penser que l'accroissement récent de l'offre dans ce domaine accélérera ce phénomène. II reste maintenant à savoir si ces services supplanteront définitivement les anciennes pratiques, basées sur l'acquisition d'albums ou de singles, en format physique ou numérique.

Grâce à cette enquête, nous disposons maintenant de données de référence sur les habitudes de consommation de musique et le comportement dans la recherche d'information musicale des Québécois âgés de 18 à 35 ans. Ces informations sont utiles non seulement pour les bibliothécaires mais aussi pour les concepteurs de services d'écoute de musique en ligne et les acteurs de l'industrie musicale. Bien que nous ayons pu relever quelques légères différences entre les résultats de notre enquête et ceux d'enquêtes réalisées auprès d'autres populations, nous constatons que les jeunes Québécois semblent suivre globalement la tendance générale. La prochaine étape consisterait à reproduire cette enquête avec un échantillon probabiliste, en l'élargissant aux autres tranches d'âge, afin de voir si nos résultats peuvent être généralisés à l'ensemble de la population québécoise.

\section{Repenser l'offre musicale en bibliothèque}

Que peuvent tirer les bibliothèques de cette enquête? Comme le démontrent nos résultats, l'entourage - les amis, les collègues, la famille - demeure la source privilégiée par les jeunes adultes pour découvrir de nouvelles musiques, suivie des services de recommandations intégrés aux plateformes de lecture de musique en continu. En contrepartie, le spécialiste en musique, malgré ses connaissances pointues, se retrouve en dernière position (voir Tableau 2). Ces résultats viennent confirmer les résultats d'études antérieures qui montraient que les gens ont rarement recours aux 
spécialistes pour découvrir de la musique, parce qu'ils leur font peu confiance ou par crainte d'être perçus comme ayant une culture musicale pauvre. Ces résultats suggèrent que le bibliothécaire qui souhaiterait offrir des services de conseils pour la musique, à l'instar des services de conseil de lectures déjà offerts dans les bibliothèques (les « readers' advisory services »), devrait prendre certaines précautions. II paraît essentiel pour le bibliothécaire de prendre soin de créer un lien de confiance avec les usagers qui sollicitent ses conseils, de se montrer accueillant et de faire attention de rester neutre vis-à-vis des goûts musicaux personnels des usagers. II devrait se présenter non pas comme un spécialiste qui sait juger ce qui est de la musique de qualité, mais plutôt comme une personne ressource pouvant guider les usagers dans leurs choix musicaux, en fonction de leurs goûts et de leurs besoins. Pour créer un lien de confiance avec les usagers, l'organisation d'ateliers de médiation ou la mise sur pied d'un cercle d'écoute pourraient s'avérer utiles.

Pour ce qui est des collections, il est aujourd'hui presque impossible pour une bibliothèque de petite ou de moyenne taille de rivaliser avec l'offre musicale gratuite sur le Web. Devant ce constat, il pourrait être tentant pour les bibliothèques de cesser d'acquérir des CD ou des DVD musicaux et ainsi de ne plus mettre à jour une offre musicale de qualité à leurs usagers. Or, comme nous l'avons vu, plusieurs personnes, y compris les jeunes adultes, restent attachées au disque. De plus, tout n'est pas disponible gratuitement sur le Web. L'offre de musique classique et de musiques traditionnelles du monde y est notamment beaucoup plus limitée. Ainsi, la bibliothèque a un rôle à jouer pour s'assurer que la communauté qu'elle sert a accès à tous les types de musique. Elle peut donc développer son offre en fournissant à sa communauté un accès à des services d'écoute de musique en ligne payants qui incluent dans leur catalogue des types de musique plus difficiles à trouver sur le Web.

\section{Bibliographie}

Cunningham, S. J., Reeves, N. et Britland, M. (2003). An ethnographic study of music information seeking: implications for the design of a music digital library. In Proceedings of the third ACM/IEEE-CS joint conference on Digital libraries (p. 516).

Ewoudou, J. (2005). Comprendre la consommation de produits et services de la culture au Canada. Statistique Canada.

Fortier, C. (2015). Les ventes d'enregistrements sonores au Québec en 2014. Bulletin Coup d'œil sociodémographique de l'Institut de la statistique du Québec, 39.

Gauthier, M.-A. (2014). L'évolution de la fréquentation scolaire au Québec depuis 1990. Bulletin Coup d'œil sociodémographique de I'Institut de la statistique du Québec, 33.

Hu, X., Lee, J. H. et Wong, L. K. Y. (2014). Music information behaviors and system preferences of university students in Hong Kong. In Proceedings of the 15th International Society for Music Information Retrieval Conference (ISMIR 2014) (p. 579-584). ISMIR. 
IFPI. (2015). IFPI digital music report 2015: Charting the path to sustainable growth.

Institut de la statistique du Québec. (2015). Répartition de la population de 25 à 64 ans selon le plus haut niveau de scolarité atteint, la région administrative, l'âge et le sexe, Québec. In Statistique Canada (SC), Enquête sur la population active, compilation spéciale, adapté par I'Institut de la statistique du Québec (ISQ).

Kamalzadeh, M., Baur, D. et Moller, T. (2012). A survey on music listening and management behaviours. In Proceedings of the 13th International Society for Music Information Retrieval Conference (ISMIR 2012). ISMIR.

Krause, A. E. et North, A. C. (2016). Music listening in everyday life: Devices, selection methods, and digital technology. Psychology of Music, 44(1), 129-147.

Laplante, A. (2008). Everyday life music information-seeking behaviour of young adults: an exploratory study. (Thèse de doctorat), McGill University, Montréal.

Laplante, A. (2010a). The role people play in adolescents' music information acquisition. In A. Anglade, C. Baccigalupo, N. Casagrande, Ò. Celma et P. Lamere (dir.), Proceedings of the Workshop on Music Recommendation and Discovery 2010 (Vol. 633) : Ceur-WS.

Laplante, A. (2010b). Users' relevance criteria in music retrieval in everyday life: An exploratory study. In J. S. Downie et R. C. Veltkamp (dir.), Proceedings of the 11th International Society for Music Information Retrieval Conference (ISMIR 2010) (p. 601-606). International Society for Music Information Retrieval.

Laplante, A. (2012). Who influence the music tastes of adolescents? A study on interpersonal influence in social networks. In C. C. S. Liem, M. Müller, S. K. Tjoa et G. Tzanetakis (dir.), Proceedings of the second international ACM workshop on Music information retrieval with user-centered and multimodal strategies ( $\mathrm{p}$. 37-42). New York : ACM.

Laplante, A. (2015). Exposer ses goûts musicaux sur Facebook : quelles conséquences sur l'e-réputation des adolescents? In C. Alcantara (dir.), E-reputation : regards croisés sur une notion émergente (p. 187-193). Paris : Gualino éditeur, Lextenso éditions.

Laplante, A. et Downie, J. S. (2006). Everyday-life music information-seeking of young adults. In R. Dannenberg, K. Lemström et A. Tindale (dir.), ISMIR 2006, 7th International Conference on Music Information Retrieval (p. 381-382). Victoria : University of Victoria.

Laplante, A. et Downie, J. S. (2011). The utilitarian and hedonic outcomes of music information seeking in everyday life. Library and Information Science Research, 33(3), 202-210.

Lee, J. H., Cho, H. et Kim, Y.-S. (2016). Users' music information needs and behaviors: Design implications for music information retrieval systems. Journal of the Association for Information Science and Technology, 67(6), 1301-1330.

Lee, J. H. et Downie, J. S. (2004). Survey of music information needs, uses, and seeking behaviours: Preliminary findings. In C. L. Buyoli et R. Loureiro (dir.), 
Proceedings of the 5th International Conference on Music Information Retrieval (p. 441-446). Barcelone : Universitat Pompeu Fabra.

Lee, J. H. et Waterman, N. M. (2012). Understanding user requirements for music information services. In F. Gouyon, P. Herrera, L. G. Martins et M. Müller (dir.), Proceedings of the 13th International Society for Music Information Retrieval Conference, ISMIR 2012 (p. 253-258). Porto : FEUP Edições.

The Nielson Company. (2016). 2015 Nielsen music Canada report.

Nordicity Group Limited. (2016). Étude 2016 de comparaison des tarifs des services de télécommunication offerts au Canada et à l'étranger. Conseil de la radiodiffusion et des télécommunications canadiennes.

Smirke, R. (2014). IFPI music report 2014: Global recorded music revenues fall 4\%, streaming and subs hit $\$ 1$ billion. Billboard Biz, 18 mars.

Tepper, S. J. et Hargittai, E. (2009). Pathways to music exploration in a digital age. Poetics, 37(3), 227-249. 